\title{
Two-Photon Induced Emission From Silver Nanoparticle Aggregates on Thin Films and in Solution.
}

\author{
Melinda Ewald, Jess M. Gunn, and Marcos Dantus \\ Department of Chemistry, Michigan State University, East Lansing, Michigan 48824
}

The coupling of incident electromagnetic radiation with surface plasmons on nanoscale dendritic metal particles has been observed to cause localization of the electromagnetic field, or 'hot spots'. This has led to observed enhancements of nonlinear optical processes, including surface-enhanced Raman scattering (SERS), second harmonic generation (SHG), and multiphoton photoemission [1]. These effects are reportedly strongest in aggregates that have a fractal nature.

We report on the nonlinear optical properties of dendritic silver nanostructures. The particles precipitated onto quartz cover slips and were spin coated with poly(vinyl alcohol) to reduce oxidation of the samples. An ultrafast laser capable of $10 \mathrm{fs}$ pulses at a repetition rate of $97 \mathrm{MHz}$ was brought into the rear port of an inverted microscope and focused with a $60 \mathrm{x} / 1.45$ NA objective to a focal spot size of $0.2 \mu \mathrm{m}$. The pulses were spectral phase corrected using MIIPS [2]. The sample was raster scanned and data was collected with a CCD camera.

A problem encountered when studying these samples is the low power threshold at which irreversible damage to the sample occurs. We have determined that at powers greater than $3 \mu \mathrm{W}$, damage to the silver nanoparticle aggregate sample occurs. Additionally, we have seen damage that spreads 10 diameters beyond the focal spot at higher powers, indicating a possible longer-distance effect (see Fig 1). Having determined the proper excitation power, we were able to reproducibly acquire data even after several scans, which has allowed us to carry out investigations into the effects of polarization and phase on emission.

Using a polarizer, we show that the polarization of the excitation beam does indeed affect the location of the excitation emission (see Fig 2). Some of the spots appeared with $+45^{\circ}$ polarization while others appeared with $-45^{\circ}$. Still others appeared with both polarizations, indicating a possible vertical polarization for those excitation spots. We are in the process of measuring the polarization of emitted light and its relation to the excitation polarization.

To determine the nature of the observed luminescence we prepared a solution of dendritic nanoparticles. The resulting spectrum as a function of power can be seen Fig 3. The emission was determined to arise from two-photon excitation as measured by the power dependence of broad luminescence. The observed oscillations in the spectrum are caused by the interference filter used to reject the laser beam.

\section{References}

[1] S. Link and M. A. El-Sayed, Annu. Rev. Phys. Chem. 54 (2003) 331.

[2] B. Xu, J. M. Gunn, J. M. Dela Cruz, V. V. Lozovoy and M. Dantus, J. Opt. Soc. Am. B. (2006) (in press) 

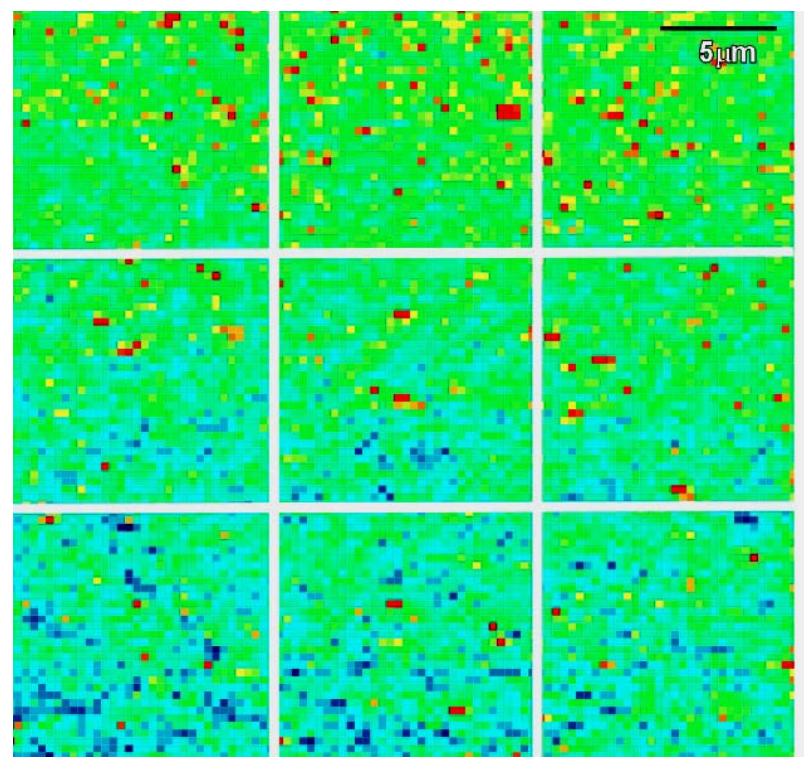
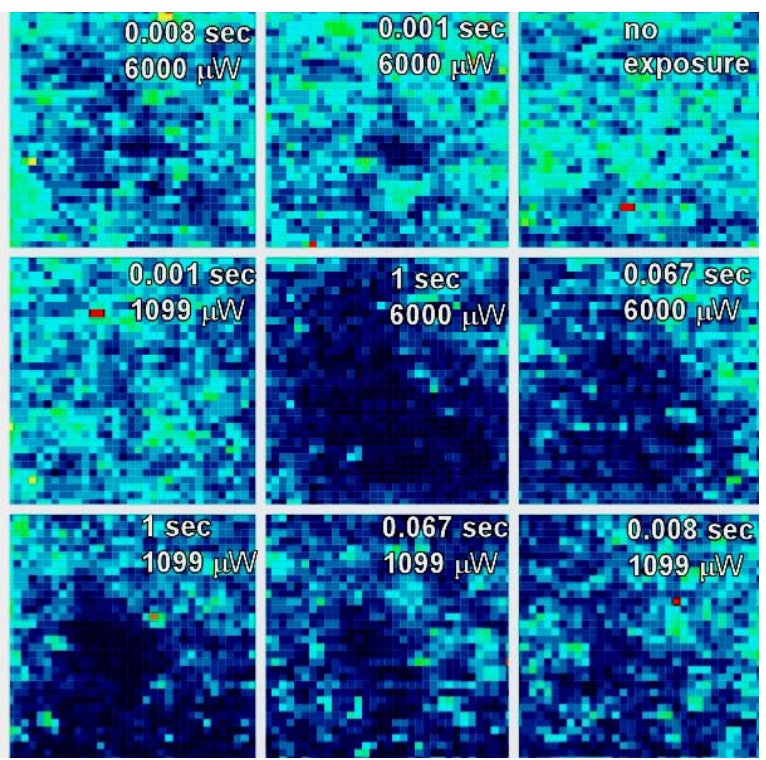

Figure 1. Laser induced damage on a thin film of dendritic silver nanoparticles. The left panel shows the first scan acquired of the region. The right panel shows the "holes" created by the power and exposure time noted for each boxed region. The incident light was focused in the center of each boxed region.

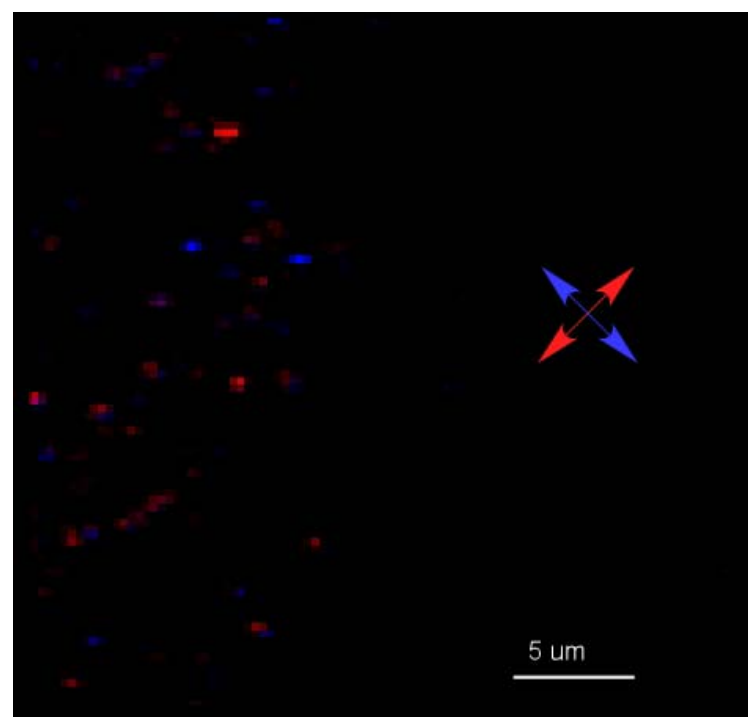

Figure 2. Effect of polarization of the excitation beam on hot spot emission. The red shows areas of emission for incoming light of $+45^{\circ}$ polarization. The blue shows areas of emission for incoming light of $-45^{\circ}$ polarization.

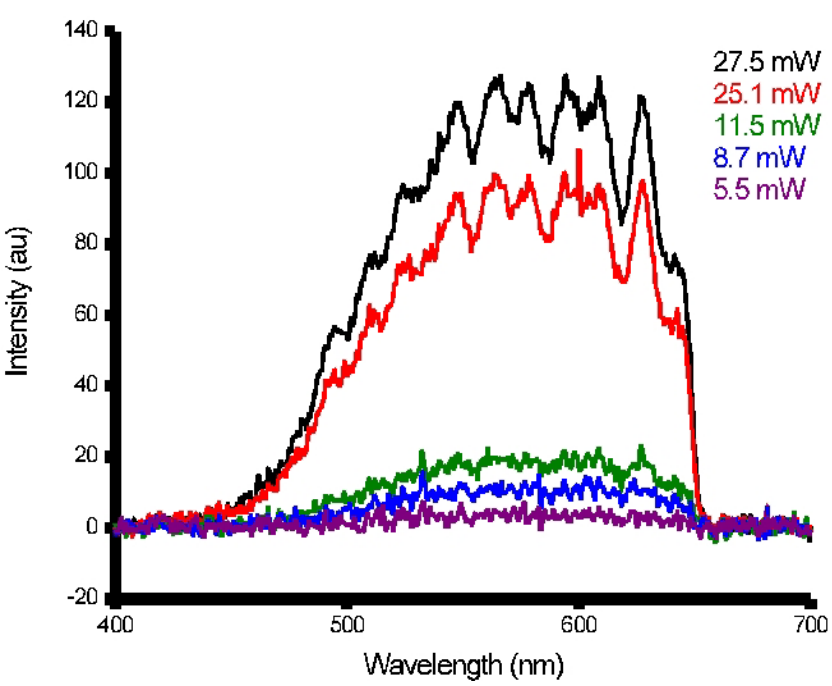

Figure 3. Quadratic laser power dependence of the broad band emission from the silver nanoparticle aggregates in solution. 